\title{
Gastrointestinal Electrical Neuromodulation for Functional Gastrointestinal Diseases, Obesity and Diabetes
}

\author{
Jieyun Yin ${ }^{1,2}$ and Jiande DZ Chen ${ }^{1,2}$ \\ ${ }^{1}$ Veterans Research and Education Foundation, VA Medical Center, Oklahoma City, Oklahoma, United States of America; and the \\ ${ }^{2}$ Division of Gastroenterology and Hepatology, Department of Medicine, Johns Hopkins University, Baltimore, Maryland, United \\ States of America
}

\begin{abstract}
Along the gut, there are rhythmic myoelectrical activities called slow waves that regulate gastrointestinal motility. Impairment in gastrointestinal slow waves and motility causes various disorders such as gastroesophageal reflux disease, gastroparesis and constipation. Gastrointestinal electrical stimulation has been introduced for the treatment of various motility diseases along the gut. Alternations in gastrointestinal motility are associated with the releases of gastrointestinal hormones, whereas changes in gastrointestinal hormones are believed to be associated with obesity and diabetes. Electrophysiology and motility of the gut are briefly reviewed. Common methods of gastrointestinal electrical stimulation are introduced. Promising or possible applications of these neuromodulation methods for treating gastroesophageal reflux disease, gastroparesis and constipation are discussed. Therapeutic potentials of electrical stimulation of the gut for the treatment of obesity and diabetes are also presented and discussed.
\end{abstract} Online address: www.bioelecmed.org doi: 10.15424/bioelectronmed.2015.00002

\section{INTRODUCTION}

Electrical stimulation of the gut was initially proposed more than half a century ago. In the earliest study, electrical stimulation was performed via an intraluminal catheter placed nasally into the duodenum, reported to enhance peristalsis of the small intestine and proposed for the treatment of ileus (1). However, follow-up studies generated controversial results, and this initial method of electrical stimulation did not mature for clinical use. One decade later in the 1970s, Kelley and colleagues developed a method of gastrointestinal pacing in which stimulation parameters were designed to alter intrinsic myoelectrical activity of the gut $(2,3)$. In this pacing method, electrical stimuli were composed of repetitive long pulses (with pulse width in the order of
50-500 ms) delivered at a frequency in the vicinity of intrinsic myoelectrical activity of the gut. However, no commercial products were made available because of high demand in energy consumption for an implantable device. In the late 1990s, an antiemetic method of gastric electrical stimulation (GES) was developed for the treatment of nausea and vomiting in patients with gastroparesis (4). This method uses short pulses delivered at a frequency much higher than the intrinsic frequency of gastric myoelectrical activity. In 2000, a method of GES, called implantable gastric stimulation (IGS), was introduced to treat obesity. It uses intermittent pulse trains with short pulse width (5). Most recently, electrical stimulation of the lower esophageal sphincter was introduced to treat gastroesophageal reflux disease

Address correspondence to Jieyun Yin, 921 NE 13th Street, VREF-151, Oklahoma City, OK 73104. Phone: 405-456-3551; E-mail: jieyunyin07@gmail.com; or Jiande DZ Chen. 4940 Eastern Ave., A-505, Baltimore, MD 21224. Phone: 410-550-6853; E-mail: jchen184@jhmi.edu. Submitted January 15, 2015; Accepted for publication January 28, 2015; Published Online (www.bioelecmed.org) January 28, 2015.

\section{The Feinstein Institute for Medical Research Empowering Imagination. Pioneering Discovery.}

(GERD) by using parameters similar to those used in nerve stimulation (6).

The aim of this article is to review major methods of gastrointestinal electrical stimulation and their potential applications and limitations. These include electrical stimulation of the lower esophageal sphincter for GERD, GES for gastroparesis (delayed emptying of the stomach), intestinal electrical stimulation (IES) for obesity and diabetes, and colon electrical stimulation (CES) for constipation.

\section{ELECTRICAL STIMULATION FOR GASTROESOPHAGEAL REFLUX}

\section{Physiology of Esophageal Motility and GERD}

Along the gut, there are myoelectrical activities that set the pace of peristalsis. However, the esophagus is an exception: no rhythmic myoelectrical activity has been reported along the esophagus. The esophagus serves as a passage for ingesting water and food. It consists of the esophageal body, and upper and lower esophageal sphincters. When ingesting food or water, the esophageal body generates peristalsis, which transports the ingested food/water to the stomach. The lower 
esophageal sphincter serves as a barrier that prevents gastric contents from entering the esophagus. When the lower esophageal sphincter is loose (low in pressure), GERD may occur and the patient may suffer from typical symptoms of heart burn.

GERD is common among general populations. Various methods have been developed for treating GERD. The most effective and commonly used medical therapy is the inhibition of gastric acid secretion using a proton pump inhibitor because the reflux of acid into the esophagus harms the esophagus. However, this method is passive, since it does not prevent reflux. Nonmedical therapies have been developed to increase the pressure of the lower esophageal sphincter by thickening or tightening the sphincter surgically, mechanically or magnetically (7). One common problem of these nonmedical methods is the side effect of dysphagia due to impaired relaxation of the sphincter during swallowing.

\section{Electrical Stimulation for GERD}

A number of electrical stimulation methods have been tested for the treatment of GERD. In one method, electrical stimulation was performed in the gastric fundus with high-frequency $(40-\mathrm{Hz})$ short pulses $(8,9)$. A significant and sustained increase in lower esophageal sphincter pressure was noted with GES in both anesthetized and conscious dogs, mediated via the cholinergic pathway. Such an effect disappeared, however, when the pulse width was increased from 0.3 to $3 \mathrm{~ms}$, suggesting a neural mechanism (8).

In a recent clinical trial, electrical stimulation was performed directly on the lower esophageal sphincter in 24 patients with GERD by using similar parameters as those used in animal GES studies (6). A significant increase in lower esophageal sphincter pressure and a significant decrease in acid reflux were noted after a 12-month treatment using an implantable device. These findings suggest a strong therapeutic potential of electrical stimulation for GERD, although more patients need to be studied and a possible placebo effect should be ruled out.

\section{GES FOR GASTROPARESIS AND OBESITY}

\section{Gastric Myoelectrical Activity and Motility}

In the stomach, there is myoelectrical activity that is composed of slow waves and spikes (10). The slow wave is a rhythmic myoelectrical event that controls the frequency and propagation of peristalsis. It is thus also called electrical control activity. The gastric slow wave originates in the proximal stomach (about one-third proximal) and propagates toward the pylorus with increasing amplitude and velocity. The frequency of gastric slow waves is about 3 cycles $/ \mathrm{min}$ (cpm) in healthy humans. The gastric slow wave is called tachygastria if its frequency is above $4 \mathrm{cpm}$ and bradygastria if its frequency is below $2 \mathrm{cpm}$. Spikes are superimposed on slow waves.

When a slow wave is superimposed with spikes, a lumen-occluded contraction occurs. Otherwise, the slow wave does not produce a gastric contraction. In the postprandial state, every slow wave is superimposed with spikes (that is, the stomach contracts at the frequency of the slow wave, which is $3 \mathrm{cpm}$ ). In the fasting state (about $4 \mathrm{~h}$ after a meal), the gastric contraction has a unique pattern called migrating motor complex (MMC) (11). The MMC migrates from the distal stomach to the distal small intestine and repeats approximately every $2 \mathrm{~h}$. Each cycle of the MMC is classified into three phases: phase I, with almost no contractions (or spikes); phase II, with slow waves intermittently superimposed with spikes; and phase III, during which each slow wave is superimposed with spikes.

\section{Pathophysiology of Gastroparesis}

Gastroparesis is defined as delayed gastric emptying of solids in the absence of mechanical obstruction (12). Itiologically, gastroparesis may be classified as diabetic, postsurgical and idiopathic. According to severity of symptoms, gastroparesis can be classified into three subgroups: mild gastroparesis (symptoms easily controlled, able to maintain weight and nutrition on a regular diet); compensated gastroparesis (moderate symptoms with only partial control with use of daily medication, able to maintain nutrition with dietary adjustments); and gastric failure (refractory symptoms that are not controlled, inability to maintain oral nutrition, emergency department visits, frequent physician visits, or hospitalization) $(13,14)$.

Main pathophysiologies of gastroparesis include visceral hypersensitivity, impaired gastric accommodation and impaired gastric motility that includes antral hypomotility and impaired coordination, gastric dysrhythmia and delayed gastric emptying. (10,15-18).

\section{Gastric Electrical Stimulation for Gastroparesis}

Different methods of GES have been proposed for the treatment of motility disorders of the stomach, and different classification methods have also been used. In one classification method, GES is classified into high frequency-short pulse (frequency: a few times higher than the frequency of gastric slow waves; pulse width below $6 \mathrm{~ms}$ ) and low frequency-long pulse methods (frequency: in the vicinity of gastric slow waves; pulse width: a few hundred milliseconds) (19). The first method is also called Enterra Therapy (Enterra ${ }^{\circledR}$ Therapy, Medtronic, Minneapolis, MN, USA) $(20,21)$. The device has received approval from the U.S. Food and Drug Administration for humanitarian use to treat nausea and vomiting in patients with gastroparesis. The improvement in nausea and vomiting with the Enterra Therapy is believed to be mediated via the central and vagal mechanisms rather than gastric motility (19). However, controversial findings have been reported; a recent meta-analysis seems to suggest mechanisms involving gastric motility, primarily gastric emptying (22). Temporary GES using intraluminal mucosal electrodes has been adopted for screening patients and improving efficacies of the therapy (23). A less invasive method of 
placing stimulation electrodes percutaneously has also been introduced for the Enterra Therapy (24).

The low frequency-long pulse GES is commonly called "gastric pacing." Gastric pacing is designed to alter gastric motility, including gastric slow waves, gastric contractions and gastric emptying. Entrainment of gastric slow waves has been reported in a number of GES studies that used long pulse stimulation (25-28). This entrainment approach to GES has been used to improve gastric dysrhythmias in dogs induced by vasopressin and glucagon $(29,30)$ and in diabetic mice with delayed gastric emptying (31). A number of innovative methods of gastric pacing have been reported, including multichannel GES (25), dual pulse GES (29) and synchronized GES $(30,31)$.

Because of the lack of commercially available devices, clinical findings are limited with the gastric pacing method. By using a custom-made external device, one- and two-channel gastric pacing has been shown to improve symptoms and gastric emptying in patients with gastroparesis (1,32). Gastric pacing methods seem more promising than the Enterra Therapy because of their prokinetic effect on gastric motility. However, currently, no commercially available device is available for gastric pacing.

\section{Gastric Electrical Stimulation for Obesity}

The rate of gastric emptying is known to be associated with the feeling of fullness and satiety; therefore, it has been suggested to be linked to obesity (33). Obesity is defined as the accumulation of excess body fat leading to a high body mass index $\left(>30 \mathrm{~kg} / \mathrm{m}^{2}\right)(33,34)$. Altered gastrointestinal motility has been reported in obesity. A recent study indicated that obese subjects showed unaltered gastric slow waves at baseline but enhanced responses to both fatty and protein meals compared with lean subjects (35). Although controversial, accelerated gastric emptying was reported in most of the studies in both animals and humans $(36,37)$.
GES was first introduced for the treatment of obesity by using trains of short pulses (IGS), with parameters as follows: pulse width of 0.3-0.5 ms and frequency of $40 \mathrm{~Hz}$ and train on-time of $2 \mathrm{~s}$ and offtime of 3 s. Open-label clinical trials reported significant weight loss in obese patients $(5,38)$; however, multicenter, double-blinded, placebo-controlled trials have failed to show significant weight loss compared to sham-GES (39). Basic laboratory research demonstrated that the failure of IGS in producing sufficient weight loss was attributed to its limited effects on the central satiety system and lack of effects on gastric motility, resulting from the use of narrow pulses (40). Most recent studies on GES with wide pulses designed to inhibit gastric motility has shown robust and significant effects on food intake and weight loss in both rats and dogs $(41,42)$. Clinical studies are needed to prove the therapeutic potential of this method for treating obesity.

On the contrary, there was another method of GES for obesity, the Tantalus system, which was designed to enhance gastric motility rather than inhibiting motility (43). Under normal physiological conditions, the gastric antrum contracts upon food intake for mixing, grading and expelling ingested food into the small intestine. This postprandial antral contractive activity activates vagal afferent nerves and sends a signal to the central satiety center. The Tantalus system was designed to reinforce postprandial antral contractions, thus enhancing postprandial satiety. An open-label study demonstrated a significant weight loss with 1 year of treatment of such a GES method (43). However, no follow-up studies are available showing similar significant weight loss effects.

\section{INTESTINAL ELECTRICAL STIMULATION FOR DIABETES}

\section{Small Intestinal Myoelectrical Activity and Motility}

Myoelectrical activity in the small intestine is also composed of slow waves and spike potentials. Like in the stom- ach, the slow wave in the small intestine is also rhythmic but with a frequency gradient along the tract: 12 cycles/min in the duodenum and 9 cycles/min in the ileum in humans (44). Spikes potentials in the small intestine are one-to-one correlated with intestinal contractions.

Similar to the stomach, the small intestinal motility also exhibits different patterns in the fasting and fed state, respectively. The MMC is the pattern of the small intestinal motility in the fasting state. As in the stomach, it repeats about every $2 \mathrm{~h}$ and divides into three phases. The role of the MMC is to transport nondigestible gastric contents from the stomach to the colon. The small intestinal contractions in the fed state are intermittent with different propagation patterns: antegrade, simultaneous and retrograde. These patterns are believed to be associated with the absorption function of the small intestine. The mixture of these patterns allows the small intestine to have sufficient time to absorb nutrients.

\section{Gastrointestinal Hormones}

Fat in the diet stimulates gastrointestinal hormone release including cholecyctokinin (CCK), peptide YY (PYY) and glucagon-like peptide-1 (GLP-1) (45). Nutrients control appetite and energy intake through two principal mechanisms: the stomach and small intestine (small intestines play a dominant role) (46). Triacylglycerides in the small intestine stimulate gastrointestinal anorectic hormone release, including CCK and PYY (47), which stimulate receptors on vagal afferents that convey information from the viscera to the central nervous system and medicate alterations in antropyloroduodenal motility and slowing of gastric emptying $(48,49)$. GLP-1 is produced by the processing of proglucagon in the enteroendocrine L cell of the intestinal mucosa. It is released in response to meal ingestion (50). GLP-1 is known to increase gastric volume during the fasting and postprandial period and retard gastric emptying (51); this inhibitory effect was involved with the vagus nerve (52). GLP-1 also exerts a glucose-dependent effect on pancreas 
cells and enhances insulin release. it also has an inhibitory effect on the pancreatic alpha cells, therefore reducing glucagon release (51). These properties of GLP-1 on gastrointestinal motility and glycemic control provide the rationale for treating obesity and diabetes.

\section{Intestinal Electrical Stimulation and Motility}

On the basis of stimulation pulse width, IES can be classified into long pulse stimulation, short pulse stimulation, pulse train stimulation, dual pulse stimulation (the stimulus is composed of one or a few short pulses followed by a long pulse) and synchronized stimulation (IES is performed at the occurrence of cyclic physiological electrical events of the small intestine) (53).

Studies have shown that IES with long pulses (pulse width $>50 \mathrm{~ms}$ ) entrains intestinal slow waves and normalizes intestinal dysrhythmia (54). Whereas intestinal slow wave dysrhythmia can be reliably and consistently improved with IES or intestinal pacing, conventional IES with long pulses has not improved intestinal contractions. It was reported that only synchronized IES had an ameliorating effect on intestinal contractions (55). On the other hand, the inhibitory effect of IES on gastrointestinal motility has been frequently reported both in animals and humans. In dogs, IES was found to reduce gastric tone, inhibit antral contractions and delay gastric emptying (56); similar inhibitory effects of IES on gastric emptying, food intake and body weight were also noted in rats (57). In humans, long pulse IES delivered via intraluminal ring electrodes attached to a feeding tube placed in the duodenum reduced gastric accommodation and delayed gastric emptying (58). IES on small intestinal motility seems to be location related. Forward IES accelerates intestinal transit (59-61), while backward IES delays intestinal transit $(62,63)$.

The IES-induced acceleration in small intestinal transit resulted in reduced small bowel absorption. IES with long pulses or pulse trains accelerated intes- tinal transit measured by the recovery of phenol red and increased the percentage of triglycerides recovered from the distal segment. The effects were partially abolished with the treatment of lidocaine, suggesting possible involvement of the enteric nerves (64). Similar findings on the acceleration in small intestinal transit and reduction in absorption were also reported in healthy volunteers with IES by using intraluminal electrodes (65).

\section{Potentials of IES for Obesity and Diabetes}

The accelerative effect of IES has led to its potential therapy for intestinal dysmotility; it may be used for treating intestinal pseudo-obstruction and postoperative ileus (53). Moreover, IES-induced alternations in gastrointestinal motility and hormones are also suggestive of its therapeutic potentials for obesity and diabetes. Intuitively, IES-induced delay in gastric emptying may lead to reduced food intake, yielding weight loss. Similarly, IES-induced acceleration in small intestinal transit may lead to reduced absorption and thus weight loss. Reduced food intake and nutrient absorption results in reduced postprandial glycemia and are thus beneficial to glycemic control in diabetes.

In diet-induced obese rats, IES was reported to delay gastric emptying and reduce food intake and weight gain (57); in pigs, similar findings were observed, and small intestinal transit was accelerated as well (35). The central mechanism is involved in the inhibitory effect of IES for obesity. It was reported that IES with either long pulses or short pulses activated the neurons in the ventromedial nucleus of the hypothalamus (VMH) (66). VMH is known to be closely related to the regulation of feeding behaviors and plays an important role in the mediation of satiety. Although the animal data are promising, to date, no clinical data are available to validate the potential treatment option of IES for obesity because of the unavailability of a suitable implantable device for IES. Similar to GES, all commercially available implantable devices are capable of generating pulses with a width of $\leq 1 \mathrm{~ms}$, whereas wider pulses are needed for IES to alter intestinal motility or transit.

The potential of IES for treating diabetes was first reported by Khawaled et al. (67). IES applied to the duodenum was shown to decrease postprandial blood glucose level with a concurrent decrease in insulin secretion in anesthetized rats. The authors believed it was possibly because of delayed gastric emptying and increased intestinal flow rate of nutrients (67). A later study by Sandoval et al. (68) indicated IES in combination with nutrient infusion significantly increased plasma GLP-1, which is known to have a hypoglycemic effect. Although the current data are preliminary, the alteration of gastrointestinal motility combined with hormonal and central regulation suggests the therapeutic potential of IES for treating diabetes. In addition to IES, GES was also shown to have a hypoglycemic effect. With the above method of the Tantalus system, researchers reported that postprandial GES in synchronization with antral slow waves resulted in a significant reduction in $\mathrm{Hb} \mathrm{A}_{1 \mathrm{c}}$ in about a dozen patients with diabetes (69). However, placebo and medication effects could not be ruled out, since the study was uncontrolled and open-label and the patients were concurrently treated with one or more antidiabetic medications.

\section{COLONIC ELECTRICAL STIMULATION FOR CONSTIPATION}

\section{Physiology of Colon Motility}

Colon motility is complex and difficult to characterize. It is featured with individual contractions and giant migrating contractions. The individual contraction can be classified as short duration (15 s) or long duration (40-60 s) and is without dominant frequencies (70). The giant migrating contractions occur only once or twice daily and are often associated with mass movement along the colon. When convenient, the giant migrating contractions could lead to defecation. 
There are slow waves in the colon. Unlike the slow waves in the stomach and small intestine, there is no dominant frequency in the slow waves in the colon. Slow waves oscillate in various frequency bands below $1 \mathrm{~Hz}$ or 60 cycles/min. Sometimes, slow waves with different frequencies are superimposed on each other. These complicated features of the colonic slow waves are the basis of the mixed patterns of colon motility that are associated with colon function (storage and water absorption).

\section{Colonic Electrical Stimulation for Constipation}

There has been considerable interest in CES, although no commercial products are available for treating constipation by using an implantable device. Two methods have been used for CES: pulse trains at a frequency of $20-50 \mathrm{~Hz}$ and repetitive long pulses (a few hundred milliseconds) at a frequency of $<1 \mathrm{~Hz}$. In rats, both methods of CES were reported to accelerate colonic transit $(71,72)$. In dogs, pulse train CES was reported to induce colon contractions facilitating mass movement (73) and was more effective than longpulse CES in accelerating colon transit (74). In a preliminary clinical study, longpulse CES was reported to improve evacuation of the colon in patients with colonic inertia (75). More studies are needed before the clinical role of CES for the treatment of constipation can be established. Mechanistically, the accelerative effect of CES on the colon was found to involve the cholinergic pathways (74).

\section{DISCUSSION AND CONCLUSIONS}

Although electrical stimulation of the gastrointestinal tract has been under investigation for more than half a century, the progress has been slow compared with other methods of electrical stimulation, such as vagal nerve stimulation, spinal cord stimulation and deep brain stimulation. In addition to being invasive, one of the other major hindrances is attributed to the fact that gastrointestinal electrical stimulation is directly applied to smooth muscles instead of nerves. Smooth muscles have a large time constant; therefore, much wider or longer pulses are needed for gastrointestinal electrical stimulation than nerve stimulation. Increases in pulse width from microsecond to millisecond dramatically increase energy consumption, making it difficult for the development and application of an implantable pulse generator. The problem can now be resolved with availability of remote charging technologies and even wireless stimulation methods.

Compared to cardiac pacing and electrical nerve stimulation, much less is known about mechanisms of gastrointestinal electrical stimulation. Different from other methods of electrical stimulation, electrical stimulation of the gut involves gastrointestinal smooth muscles and the enteric nervous system in addition to autonomic and central mechanisms. Most of the previous studies investigated the effects of gastrointestinal electrical stimulation on gastrointestinal motility and symptoms; little is known about the cellular mechanisms involving smooth muscles or neural mechanisms involving enteric nerves. More mechanistic studies are necessary to improve methodologies of gastrointestinal electrical stimulation and bring more technologies to the bedside.

In summary, electrical stimulation of the lower esophageal sphincter has been shown to be effective in treating GERD by elevating the pressure of the sphincter. More clinical data are needed, and placebo-controlled studies are also necessary. In the esophagus, there is a need to improve esophageal peristalsis because impaired esophageal peristalsis may cause dysphagia and worsen GERD (impaired clearance of refluxed acid in the esophagus). It is yet to be determined whether sequential electrical stimulation along the esophageal generates peristalsis. Electrical stimulation of the stomach is attractive and has been studied most extensively. It seems that GES with short pulses (Enterra Therapy) is capable of improving nausea and vomiting in patients with gastroparesis; it may also be appli- cable to treat nausea and vomiting due to other causes. Gastric pacing (use of long pulses) with appropriate parameters is able to improve gastric emptying and thus gastroparesis; however, there is a need to develop a new generation of implantable pulse generator. IES has a great potential for treating obesity and diabetes based on laboratory research. Clinical studies are needed to prove these therapeutic potentials. Colon electrical stimulation may be applied for treating constipation based on promising animal and clinical studies. Physiologically, CES may be more difficult to design than GES or IES because of the complexity of colonic electrophysiology and physiology of colon motility. Clinical studies are needed to establish its role in treating patients with colonic motility disorders.

\section{DISCLOSURE}

The authors declare that they have no competing interests as defined by Bioelectronic Medicine, or other interests that might be perceived to influence the results and discussion reported in this paper.

\section{REFERENCES}

1. McCallum RW, et al. (1998). Gastric pacing improves emptying and symptoms in patients with gastroparesis. Gastroenterology. 114:456-61.

2. Kelly KA, La Force RC. (1972) Pacing the canine stomach with electric stimulation. Am. J. Physiol. 222:588-94.

3. Kelly KA, Code CF. (1977) Duodenal-gastric reflux and slowed gastric emptying by electrical pacing of the canine duodenal pacesetter potential. Gastroenterology. 72:429-33.

4. Abell TL, et al. (2002) Gastric electrical stimulation in intractable symptomatic gastroparesis. $\mathrm{Di}$ gestion. 66:204-12.

5. Cigaina V. (2002) Gastric pacing as therapy for morbid obesity: preliminary results. Obes. Surg. 12(1 Suppl):12S-16S.

6. Rodríguez L, et al. (2013) Long-term results of electrical stimulation of the lower esophageal sphincter for the treatment of gastroesophageal reflux disease. Endoscopy. 45:595-604.

7. Perry KA, et al. (2015) 2014 SSAT State-of-the-Art Conference: Advances in Diagnosis and Management of Gastroesophageal Reflux Disease. J. Gastrointest. Surg. 19:458-66.

8. Xing JH, Lei Y, Chen JD. (2005) Gastric electrical stimulation (GES) with parameters for morbid obesity elevates lower esophageal sphincter (LES) pressure in conscious dogs. Obes. Surg. 15:1321-7. 
9. Xing J, Felsher J, Brody F, Soffer E. (2005) Gastric electrical stimulation significantly increases canine lower esophageal sphincter pressure. Dig. Dis. Sci. 50:1481-7.

10. Chen JD, Pan J, McCallum RW. (1993) Clinical significance of gastric myoelectrical dysrhythmias. Dig. Dis. 13:275-90.

11. Yamata T, Alpers DH, Owyang C, Powell DW, Sliverstein FE. (1995) Textbook of Gastroenterology. Philadelphia, PA: JB Lippincott Company.

12. Abell TL, et al. (2006) Treatment of gastroparesis: a multidisciplinary clinical review. Neurogastroenterol. Motil. 18:263-83.

13. Parkman HP, et al. (2010) Gastroparesis and functional dyspepsia: excerpts from the AGA/ANMS meeting. Neurogastroenterol. Motil. 22:113-33.

14. Stanghellini V, Tack J. (2014) Gastroparesis: separate entity or just a part of dyspepsia? Gut. 63:1972-8.

15. Malagelada JR, Rees WD, Mazzotta LJ, Go VL. (1980) Gastric motor abnormalities in diabetic and postvagotomy gastroparesis: effect of metoclopramide and bethanechol. Gastroenterology. 78:286-93

16. Tack J (2007). Gastric motor disorders. Best Pract. Res. Clin. Gastroenterol. 21:633-44.

17. Tack J, Piessevaux H, Coulie B, Caenepeel P, Janssens J. (1998) Role of impaired gastric accommodation to a meal in functional dyspepsia. Gastroenterology. 115:1346-52.

18. Tack J, et al. (2006) Functional gastroduodenal disorders. Gastroenterology. 130:1466-79.

19. Yin J, Abell TD, McCallum RW, Chen JD. (2012) Gastric neuromodulation with Enterra system for nausea and vomiting in patients with gastroparesis. Neuromodulation. 15:224-31.

20. Familoni BO, Abell TL, Nemoto D, Voeller G, Johnson B. (1997) Efficacy of electrical stimulation at frequencies higher than basal rate in canine stomach. Dig. Dis. Sci. 42:892-7.

21. Abell T, et al. (2003) Gastric electrical stimulation for gastroparesis improves nutritional parameters at short, intermediate, and long-term followup. J. Parenter. Enteral. Nutr. 27:277-81.

22. O'Grady G, et al. (2009) High-frequency gastric electrical stimulation for the treatment of gastroparesis: a meta-analysis. World J. Surg. 33:1693-701.

23. Abell TL, et al. (2011) A double-masked, randomized, placebo-controlled trial of temporary endoscopic mucosal gastric electrical stimulation for gastroparesis. Gastrointest. Endosc. 74:496-503.

24. Elfvin A, et al. (2007) Percutaneous implantation of gastric electrodes: a novel technique applied in animals and in patients. Neurogastroenterol. Motil. 19:103-9.

25. Chen JD, et al. (2005) Efficiency and efficacy of multi-channel gastric electrical stimulation. $\mathrm{Neu}$ rogastroenterol. Motil. 17:878-82.

26. Song G, et al. (2005) Two-channel gastric electrical stimulation accelerates delayed gastric emptying induced by vasopressin. Dig. Dis. Sci. 50:662-8.

27. Lin Z, Forster J, Sarosiek I, McCallum RW. (2003) Treatment of gastroparesis with electrical stimulation. Dig. Dis. Sci. 48:837-48.
28. Bortolotti M. (2002) The "electrical way" to cure gastroparesis. Am. J. Gastroenterol. 97:1874-83.

29. Liu J, Qiao X, Chen JD. (2006) Therapeutic potentials of a novel method of dual-pulse gastric electrical stimulation for gastric dysrhythmia and symptoms of nausea and vomiting. Am. J. Surg. 191:255-61.

30. Zhu H, Sallam H, Chen DD, Chen JD. (2007) Therapeutic potential of synchronized gastric electrical stimulation for gastroparesis: enhanced gastric motility in dogs. Am. J. Physiol. Regul. Integr. Comp. Physiol. 293:R1875-81.

31. Song GQ, Chen JD. (2007) Synchronized gastric electrical stimulation improves delayed gastric emptying in nonobese mice with diabetic gastroparesis. J. Appl. Physiol. 103:1560-4.

32. Lin Z, et al. (2011) Two-channel gastric pacing in patients with diabetic gastroparesis. Neurogastroenterol. Motil. 23:912-e396.

33. Mushref MA, Srinivasan S. (2013) Effect of high fat-diet and obesity on gastrointestinal motility. Ann. Transl. Med. 1:14

34. Xing J, Chen JD. (2004) Alterations of gastrointestinal motility in obesity. Obes. Res. 12:1723-3.

35. Xu X, Lei Y, Chen JD. (2011) Duodenum electrical stimulation delays gastric emptying, reduces food intake and accelerates small bowel transit in pigs. Obesity (Silver Spring). 19:442-8.

36. Zhang J, Sha W, Zhu H, Chen JD. (2013) Blunted peripheral and central responses to gastric mechanical and electrical stimulations in diet-induced obese rats. J. Neurogastroenterol. Motil. 19:454-66.

37. Wright RA, Krinsky S, Fleeman C, Trujillo J, Teague E (1983) Gastric emptying and obesity. Gastroenterology. 84:747-51.

38. D'Argent J. (2012) Gastric electrical stimulation as therapy of morbid obesity: preliminary results from the French study. Obes. Surg. 12 (Suppl. 1):21S-25S.

39. Shikora SA, et al. (2009) Implantable gastric stimulation for the treatment of clinically severe obesity: results of the SHAPE trial. Surg. Obes. Relat. Dis. 5:31-37.

40. Zhang J, Tang M, Chen JD. (2009) Gastric electrical stimulation for obesity: the need for a new device using wider pulses. Obesity (Silver Spring). 17:474-80.

41. Li S, Maude-Griffin R, Sun Y, Starkebaum W, Chen JD. (2013) Food intake and body weight responses to intermittent vs. continuous gastric electrical stimulation in diet-induced obese rats. Obes. Surg. 23:71-9.

42. Song GQ, et al. (2015) Gastric electrical stimulation optimized to inhibit gastric motility reduces food intake in dogs. Obes. Surg. 25:1047-55.

43. Bohdjalian A, et al. (2006) One-year experience with Tantalus: a new surgical approach to treat morbid obesity. Obes. Surg. 16:627-34.

44. Christensen J, Schedl HP, Clifton JA. (1966) The small intestinal basic electrical rhythm (slow wave) frequency gradient in normal men and in patients with variety of diseases. Gastroenterology. 50:309-15.

45. Stewart JE, Feinle-Bisset C, Keast RS. (2011) Fatty acid detection during food consumption and digestion: associations with ingestive behavior and obesity. Prog. Lipid Res. 50:225-33.

46. Feltrin KL, et al. (2004) Effects of intraduodenal fatty acids on appetite, antropyloroduodenal motility, and plasma CCK and GLP-1 in humans vary with their chain length. Am. J. Physiol. Regul. Integr. Comp. Physiol. 287:R524-33.

47. Little TJ, et al. (2007) Free fatty acids have more potent effects on gastric emptying, gut hormones, and appetite than triacylglycerides. Gastroenterology. 133:1124-31.

48. Berthoud HR. (2008) Vagal and hormonal gut-brain communication: from satiation to satisfaction. Neurogastroenterol. Motil. 20 (Suppl. 1):64-72.

49. Feltrin KL, et al. (2008) Comparative effects of intraduodenal infusions of lauric and oleic acids on antropyloroduodenal motility, plasma cholecystokinin and peptide $\mathrm{YY}$, appetite, and energy intake in healthy men. Am. J. Clin. Nutr. 87:1181-7.

50. Goke R, Fehmann HC, Goke B. (1991) Glucagonlike peptide-1(7-36) amide is a new incretin/ enterogastrone candidate. Eur. J. Clin. Invest. 21:135-44.

51. Delgado-Aros S, et al. (2002) Effect of GLP-1 on gastric volume, emptying, maximum volume ingested, and postprandial symptoms in humans. Am. J. Physiol. Gastrointest. Liver Physiol. 282:G424-31.

52. Delgado-Aros S, et al. (2003) Effects of glucagonlike peptide-1 and feeding on gastric volumes in diabetes mellitus with cardio-vagal dysfunction. Neurogastroenterol. Motil. 15:435-43.

53. Yin J, Chen JD. (2010) Mechanisms and potential applications of intestinal electrical stimulation. Dig. Dis. Sci. 55:1208-20.

54. Sun Y, Song GQ, Yin J, Lei Y, Chen JD. (2009) Effects and mechanisms of gastrointestinal electrical stimulation on slow waves: a systematic canine study. Am. J. Physiol. Regul. Integr. Comp. Physiol. 297:R1392-9.

55. Yin J, Chen JDZ. (2007) Excitatory effects of synchronized intestinal electrical stimulation on small intestinal motility in dogs. Am. J. Physiol. Gastrointest. Liver Physiol. 293:G1190-5.

56. Zhao X, et al. (2009) Inhibitory effects and mechanisms of intestinal electrical stimulation on gastric tone, antral contractions, pyloric tone, and gastric emptying in dogs. Am. J. Physiol. Regul. Integr. Comp. Physiol. 296:R36-42.

57. Yin J, Zhang J, Chen JD. (2007) Inhibitory effects of intestinal electrical stimulation on food intake, weight loss and gastric emptying in rats. Am. J. Physiol. Regul. Integr. Comp. Physiol. 293:R78-82.

58. Liu S, Hou X, Chen JD. (2005) Therapeutic potential of duodenal electrical stimulation for obesity: acute effects on gastric emptying and water intake. Am. J. Gastroenterol. 100:792-6.

59. Soper NJ, Geisler KL, Sarr MG, Kelly KA, Zinsmeister AR. (1990) Regulation of canine jejunal transit. Am. J. Physiol. 259:G928-33.

60. Chen JD, Lin HC. (2003) Electrical pacing accelerates intestinal transit slowed by fat-induced ileal brake. Dig. Dis. Sci. 48:251-6. 
61. Liu J, Qiao X, Hou X, Chen JD. (2009) Effect of intestinal pacing on small bowel transit and nutrient absorption in healthy volunteers. Obes. Surg. 19:196-201.

62. Hoepfner MT, Kelly KA, Sarr MG. (1988) Pacing the canine ileostomy. Surgery. 104:476-81.

63. Sawchuk A, et al. (1986) Reverse electrical pacing improves intestinal absorption and transit time. Surgery. 100:454-60.

64. Sun Y, Chen J. (2004) Intestinal electric stimulation decreases fat absorption in rats: Therapeutic potential for obesity. Obes. Res. 12:1235-42.

65. Liu J, Xiang Y, Qiao X, Dai Y, Chen JD. (2011)

Hypoglycemic effects of intraluminal intestinal electrical stimulation in healthy volunteers. Obes. Surg. 21:224-30.

66. Zhang J, Zhu H, Chen JD. (2009) Central neuronal mechanisms of intestinal electrical stimulation: effects on duodenum distention-responsive (DD-R) neurons in the VMH of rats. Neurosci. Lett. 457:27-31.

67. Khawaled R, Blumen G, Fabricant G, Ben-Arie J, Shikora S. (2009) Intestinal electrical stimulation decreases postprandial blood glucose levels in rats. Surg. Obes. Relat. Dis. 5:692-7.

68. Sandoval D, Dunki-Jacobs A, Sorrell J, Seeley RJ, D'Alessio DD. (2013) Impact of intestinal electrical stimulation on nutrient-induced GLP-1 secretion in vivo. Neurogastroenterol. Motil. 25:700-5.

69. Sanmiguel CP, et al. (2009) Gastric electrical stimulation with the TANTALUS System in obese type 2 diabetes patients: effect on weight and glycemic control. J. Diabetes Sci. Technol. 3:964-70.

70. Sarna SKC, Cowles RV. (1984) Colonic migrating and nonmigrating motor complexes in dogs. Am. J. Physiol. Gastrointest. Liver Physiol. 246:G355-60.

71. Bruninga K, Riedy L, Keshavarzian A, Walter J. (1998) The effect of electrical stimulation on colonic transit following spinal cord injury in cats. Spinal Cord. 36:847-53.

72. Liu S, Chen JD. (2006) Colonic electrical stimulation regulates colonic transit via the nitrergic pathway in rats. Dig. Dis. Sci. 51:502-5.

73. Sanmiguel CP, Casillas S, Senagore A, Mintchev MP, Soffer EE. (2006) Neural gastrointestinal electrical stimulation enhances colonic motility in a chronic canine model of delayed colonic transit. Neurogastroenterol. Motil. 18:647-53.

74. Sallam HS, Chen JD. (2013) Colonic electrical stimulation: potential use for treatment of delayed colonic transit. Colorectal Dis. 15:e244-9.

75. Shafik A, Shafik AA, El-Sibai O, Ahmed I. (2004) Colonic pacing: a therapeutic option for the treatment of constipation due to total colonic inertia. Arch. Surg. 139:775-9.

Cite this article as: Yin J, Chen JDZ. (2015) Gastrointestinal electrical neuromodulation for functional gastrointestinal diseases, obesity and diabetes. Bioelectron. Med. 2:13-19. 